\title{
The Quantification of Operational Reliability of Agricultural Tractors with the Competing Risks Method
}

\author{
Karol DURCZAK*, Jaroslaw SELECH
}

\begin{abstract}
Reliability is one of basic parameters affecting the decision to purchase a new or used farming machine. However, there are no objective rankings of the probability of farming machine failures after any period of operation. This article presents an innovative method of quantifying the reliability of farm tractors. The method is so universal that it can also be applied to other farm vehicles and machines. Bernoulli's competing risks method enables precise indication of the right probability distribution (exponential or Weibull) on the basis of the number of identified random and age-related failures. The method was verified logically and empirically. The validation was based on the data collected from long-term users of farm tractors in Poland. Massey Ferguson tractors were found to have the best relative reliability index. This result justified the high sales of Massey Ferguson products in Europe. According to the data for 2020, they were ranked in the top three in Lithuania, Norway, and Sweden.
\end{abstract}

Keywords: Bernoulli's model; Competing risks method; Decision-making; Operations research; Tractor reliability

\section{INTRODUCTION}

The reliability theory includes several types of probability distributions, which enable the quantification of reliability of technical devices. The most common are one-parameter exponential and Rayleigh distributions, and two-parameter Weibull, normal, gamma and Poisson distributions [1-5]. Three-parameter probability distributions, e.g. Weibull and Erlang distributions are less common. The biggest problem is to select the right distribution to match the specificity of a particular technical device. A similar problem occurs when rating the reliability of agricultural machinery and vehicles which work in dynamically changing and different environmental conditions and they operate under imposed, usually very short, agrotechnical deadlines. Like most technical facilities, they are subject to both random (sudden, incidental, accidental, stochastic) and age-related (gradual) risks (failures). Therefore, we can assume that the competing risks method can be used to quantify their reliability, because it enables precise indication of the right statistical distribution.

As early as 1766 the competing risks theory was presented by Daniel Bernoulli a Swiss mathematician, who analysed the influence of smallpox on the mortality rate in Europe in the $18^{\text {th }}$ century. It was one of the first examples of modelling in epidemiology [6]. Bernoulli focused on increasing the average life expectancy by eliminating the deadly disease. He attempted to answer the question about the effectiveness of vaccination in a mathematical way [7, 8].

Since that experiment there have been numerous studies developing the theory mathematically and a wide range of publications with examples of competing risks models (CRM), e.g. in technical systems for predicting the duration of operability of technical devices [9-15].

The competing-risks model is useful in settings in which individuals (or units) may die (or fail) because of various causes. It can also be the case that for some of the items, the cause of failure is known only up to a subgroup of all causes, in which case we say that the failure is groupmasked [16].
Crowder M. J. [17] states that when trying to indicate the events that resulted in the loss of operability, it may be difficult to identify the causes. A minimum of $p$ various causes of the loss of operability is taken into account for a technical device that consists of a specific number of $m$ elements connected in series. When considering the situation in which a technical device may fail to operate due to random or age-related causes, the $(t, C)$ pair can be observed, where $t>0$ is the time of failure, whereas $C \in$ $\{1,2, \ldots, p\}$ represents the cause or type of failure. Therefore, it is assumed that there are different types of failures and each failure can be assigned to exactly one of the $p$-types. The failure time $t$ is assumed to be a continuous variable, whereas the cause $C$ may be one of the set (small) numbers $1, \ldots, p$. Thus, a two-dimensional distribution with a discrete component $(C)$ and a continuous one $(t)$ is a basic probability structure. It is assumed that one and only one cause from the given set of $p$ causes can be assigned to each failure.

It is noteworthy that failure is used as a general term. In practice, it may refer to any event of interest, depending on the technical device under study. In this case, failure does not necessarily mean the transition from the condition of operability into inoperability. Apart from that, time $t$ does not have to be interpreted as calendar time, but it can be expressed with any unit, such as the operability time, the number of cycles, mileage, etc. [8].

For example, in medicine, $C$ could be the cause of death, whereas $t$ could be the patient's age at the moment of death. In terms of reliability, $C$ could be identified as a faulty component in the system, whereas $t$ could be identified as the operating time from the start-up to the failure. In engineering our subject is more likely to be referred to as the reliability of non-repairable serial systems. In economics $t$ could be the time one has spent as a registered unemployed person and $C$ could be the cause of being deregistered. In production $t$ could be the wear (e.g. mileage) and $C$ could be the cause of failure of a machine (e.g. vehicle).

The use of competing risks in reliability tests makes it possible to include not only different causes of failures, but also different distributions of the probability of occurrence of damage for a particular type of failure. Reference 
publications provide a wide range of practical solutions which use competing risks. For example, [17] tested the tensile strength of cables and analysed two types of damage $C=2$ and tear at integrated ends or on an unconnected fragment of the cable. Mendenhall and Hader studied very high frequency (VHF) radio transmitters and receivers [18]. They presented data on the time of occurrence of two types of failures: those confirmed at the service centre and those unconfirmed.

Contemporary reliability databases distinguish various types of failures. They are categorised as critical failures, which make further operation impossible, failures which may require a repair of a faulty item and initial failures. Traditionally, competing risks are considered as if they were independent of each other. However, this assumption may be incorrect in some cases, for example, when we consider common factors such as the working or production environment, etc. [19].

The competing risks method may help to correctly set the frequency of overhauls or replacement of parts before damage occurs. This action may be taken to significantly reduce the operating costs. Competing risks often consider only two types of failures: sudden failures and failures resulting from reduced functional characteristics.

The aim of the study was to develop a simplified method of evaluation of the reliability of farm tractors on the basis of the number of random and age-related failures which occurred during a specific period of their operation. This method will enable the creation of relevant reliability rankings for farm tractor users, manufacturers, traders and servicemen.

\section{MATERIALS AND METHOD}

Farm tractors were the subject of reliability tests. They are basic energy machines on every mechanised farm, regardless of its production profile. There are about 1.5 million farm tractors registered in Poland. In terms of statistics this means that every Polish farm owns at least one tractor. Their average annual operation time is about 600 hours of operation (in Germany, up to 1000 hours of operation). Therefore, it is necessary for potential customers to carefully consider their purchase decisions. For this reason, it is recommended to do all the work to facilitate rational purchase decisions to minimise uncertainty and risk. Objective brand reliability $R_{B}(t)$ rankings can be used as analytical tools for this purpose. They are based on the following algorithm (Fig. 1).

The tractor is treated holistically here, i.e. as a set of serially connected parts and systems. When one of the elements of this chain fails, the tractor becomes unusable. It does not matter whether it is a puncture or failure of an electronic system controlling the engine. Any failure causes the need to repair the tractor and renders it inoperable. It is important that the user should arbitrarily and objectively identify the failure and classify it into one of the two groups. However, sometimes the classification may be ambiguous.

The method involves several stages. First, experts decompose the technical device into a set of systems and components. The decomposition must be complete there must be no elements that do not belong to any of the components or elements belonging to several components at the same time. This stage is only ancillary to the second stage of the quantification of the reliability of farm tractors. It only helps to complete the questionnaire and can be skipped if the user has comprehensive service history of the tractor. The tractor maintenance log should contain all data that are necessary for the next stage of the procedure.

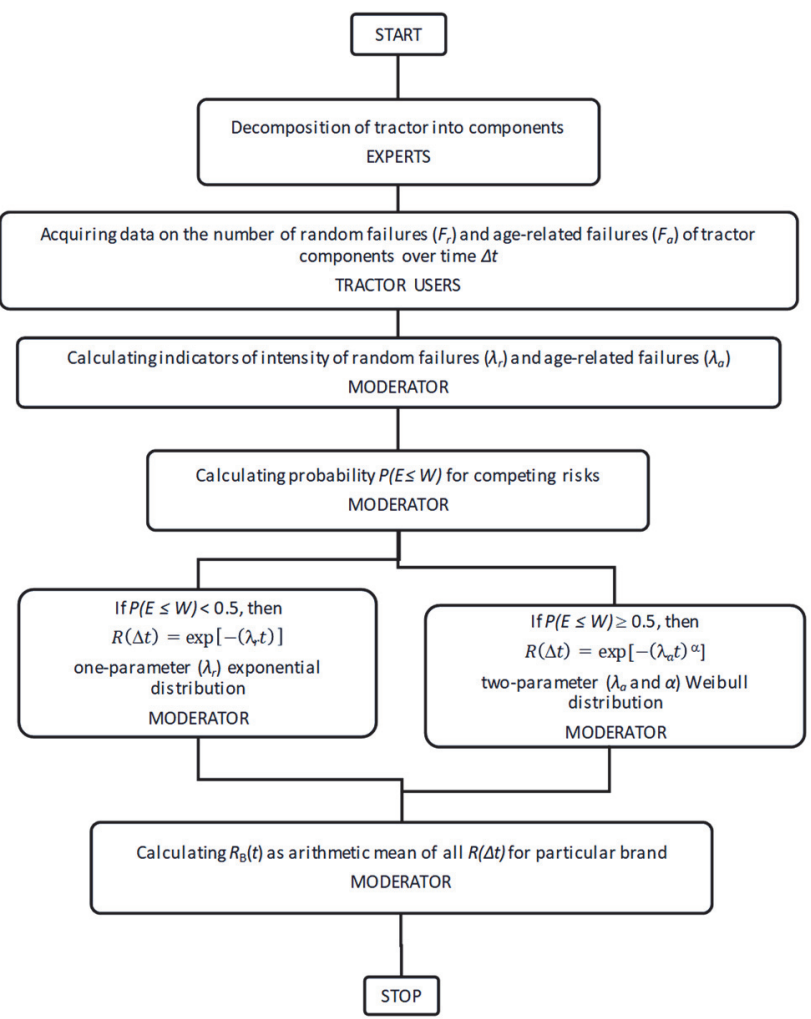

Figure $1 \mathrm{An}$ algorithm of quantification of the index of relative reliability of farm tractors $R_{B}(t)$ with the competing risks method

At this stage it is necessary to acquire reliable information from users about the number of failures of tractor components. They should be divided into random failures $F_{r}$ and age-related failures $F_{a}$ which occurred over a specific period of operation $\Delta t$ (hours of operation), where $\Delta t=t_{2}-t_{1}$ is the real time of operation of a tractor since its purchase $\left(t_{1}\right)$ until the transmission of data $\left(t_{2}\right)$ on the Internet. The data will be used to calculate the value of failure intensity indicators $\lambda_{r}$ and $\lambda_{a}$ over a period of time $\Delta t$ :

$\lambda_{r}=\frac{F_{r}}{\Delta t}$

$\lambda_{a}=\frac{F_{a}}{\Delta t}$

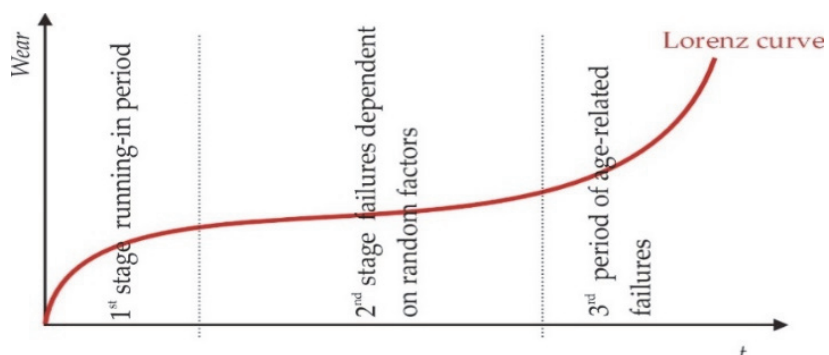

Figure 2 A classic curve showing the wear of a technical device 
The intensity of random failures $\lambda_{r}$ is approximately constant in time and does not depend on the wear of the operational potential of the technical device, according to the middle part ( $2^{\text {nd }}$ stage) of the Lorenz curve (Fig. 2).

Age-related failures $\lambda_{a}$ are caused by natural gradual wear of technical devices. The intensity of these failures increases quite rapidly at the $3^{\text {rd }}$ stage. Therefore, the form factor in the Weibull distribution must be $\alpha>1$.

At the next stage the moderator calculates the probability values for competing risks $P(E \leq W)$, where the essence of this method is to calculate the probability that the loss of operability of the system was caused by random events 1 [9]:

$P(E \leq W)=\frac{\lambda_{r}}{\lambda_{a}} \frac{\sqrt{\pi}}{2} \exp \left(\frac{\lambda_{r}}{4 \lambda_{a}^{2}}\right) \operatorname{erfc}$

where:

$\lambda_{r}$ - the intensity of random failures (events)

$\lambda_{a}$ - the intensity of age-related failures

$\exp (x)$ - exponential function

$\operatorname{erfc}(x)$ - complementary Gauss error function

$E$ - random variable with exponential distribution

$W$ - random variable with Weibull distribution.

The complementary Gauss function is used in probability theory and statistics. It is closely related with the Gauss error function $\operatorname{erfc}(x)$. The following dependence needs to be used to calculate the value of the function:

$\operatorname{erfc}(x)=\frac{2}{\sqrt{\pi}} \int_{\mathrm{x}}^{¥} \exp \left(-t^{2}\right) \mathrm{d} t$

or its value can be found in mathematical tables for the positive values of $x$. The greater the probability value $P(E$ $\leq W$ ), the more dominant the random failures become. This causes the need to use the exponential distribution. These considerations let us suppose that if we use the number of random and age-related failures in Eq. (3), it will be possible to estimate the right type of statistical distribution to quantify the operational reliability of farm tractors.

The penultimate step consists in applying the optimal statistical distribution, adequately to the specificity of failures of a particular tractor and calculating the reliability index $R(\Delta t)$ for it. The position in the final brand ranking $R_{B}(t)$ is determined by the simple arithmetic mean of all $R(\Delta t)$ models of a particular brand and an arbitrarily determined time $t$.

The method is based on the following assumptions: 1. Failure is understood as the transition of a technical facility from the condition of operability into inoperability. In this particular case, a damaged farm tractor cannot be used for the tasks it should normally do on a farm.

2. Data on failures of tractors are acquired directly from their long-term users, according to the rules 'trust the crowd' and 'believe the wisdom of the crowd', because 'experts are often wrong' [20].

3 . If the user purchased a pre-owned tractor whose meter shows $t_{1}$, but knows the complete service history of this tractor, then $\Delta t=t_{2}$.

4. The average $R_{B}(t)$ indicator can be calculated for $n$ - tractors of a particular brand on the condition that $n \geq 3$ (preferably $n \geq 30$ a large random sample).

5. 0.50 , i.e. $50 \%$ is an arbitrarily assumed value of the probability threshold $P(E \leq W)$ that determines the reliability distribution. If the value of this probability is within the interval of $(0 ; 0.50)$, the Weibull distribution with parameters $\lambda_{a}$ and $\alpha$ is used, which is logically correct. This means that age-related failures are predominant, according to Eq. (3) and Fig. 2. However, when $P(E \leq W) \in$ $<0.50 ; 1)$, the exponential distribution is used, with parameter $\lambda_{r}$ for the calculation of the operational reliability $R(\Delta t)$ of a particular tractor.

6. Random failures are treated here much more strictly than normal failures caused by material fatigue due to long-term operation. Therefore, the exponential distribution is used for them. This distribution is very often used as the most catastrophic and absolute one in the reliability theory. The curve $R(t)$ drops very steeply along with time $t$. Thus, the exponential distribution is more unfavorable than the Weibull distribution for any $\alpha$ parameter. This assumption is fully justified. Random failures result from either design errors (manufacturer's error) or users' errors (even the errors users make because of their unawareness).

7. If $F_{a}=0$ (which results in $\lambda_{a}=0$ ), there are no agerelated failures. There may only be random failures. Therefore, the exponential distribution should be applied. Thus, the error of dividing by zero in Eq. (3) can be avoided. If there are no random failures $\left(F_{r}=0\right), \lambda_{r}=0$ and the Weibull distribution can be used a priori.

8. A form factor $\alpha=1.2$ was arbitrarily assumed for the two-parameter Weibull distribution $\left(\lambda_{a}, \alpha\right)$.

9. The values of the intensity of random $\lambda_{r}$ and agerelated failures $\lambda_{a}$ are independent of each other. This means that one failure cannot be characterized as random and age-related at the same time.

Logical verification of the method:

1. The values of reliability indicators $R(\Delta t)$ and $R_{B}(t)$ as probabilities are always within the range of $0-1$, depending on the value of the failure intensity coefficients. The lack of any random or age-related failures always results in the indicator value being $R=1$.

2. If the failure intensity increases $(\lambda \uparrow)$, the value of reliability indicators goes to zero very quickly.

\section{RESULTS}

The information about the number of random and agerelated failures of farm tractors used by Polish farmers was acquired in 2019 (Fig. 3).

Respondents filled in a questionnaire on the IFOP website (Independent Farmers' Opinion Poll), which is an integral part of the website of the Department of Biosystems Engineering, Poznań University of Life Sciences, Poland [22].

It is a multi-criteria system that will allow a comprehensive assessment of the quality of agricultural machinery and vehicles. This approach is in line with the American multi-criteria decision making. $M C D M$ (or multiple-criteria decision analysis $M C D A$ ) is a subdiscipline of operations research that explicitly evaluates multiple conflicting criteria in decision making [23-29]. 
Within nine months of the operation of the website with the questionnaire survey respondents provided 47 questionnaires, but only 27 of them were used for further analysis. The ones that were rejected were either incomplete or did not meet assumption No. 4. Tab. 1 shows basic data from the questionnaires that were completed correctly.

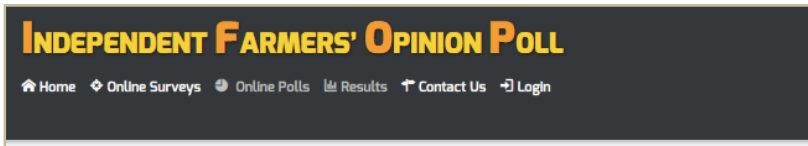

QUALITY EVALUATION OF TRACTORS - 1ST EDITION

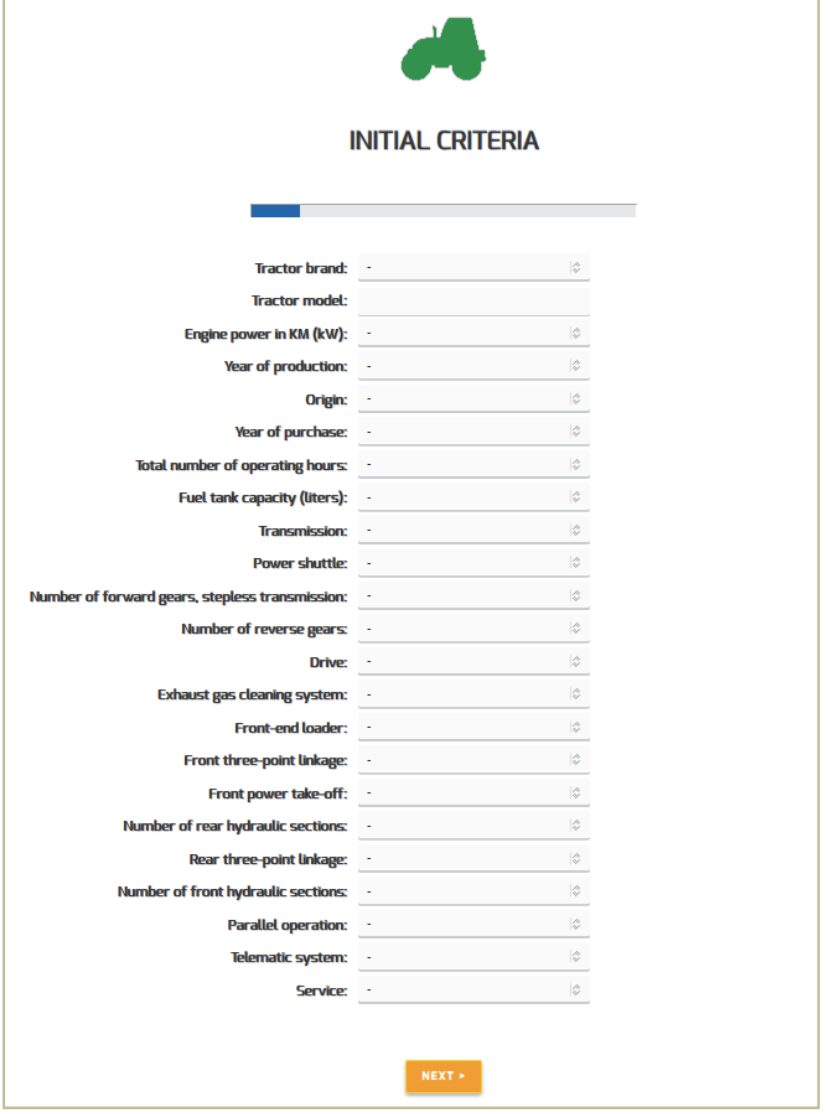

Figure 3 The home page of the questionnaire survey designed by Jurek [21]

The data were necessary to calculate indicators $R(\Delta t)$ and $R_{B}(t)$.
The knowledge of the type of distribution ( $E$ exponential or $W$ - Weibull) and the intensity of failures (column 6 or 7 ) of each of the tractors under analysis was used to calculate the average $R_{B}(t)$ values for 7 brands, where: Belarus - Tractor 1, Zetor - Tractor 2, Fendt Tractor 3, Deutz-Fahr - Tractor 4, Case IH - Tractor 5, New Holland - Tractor 6, Massey Ferguson - Tractor 7 (Fig. 4).

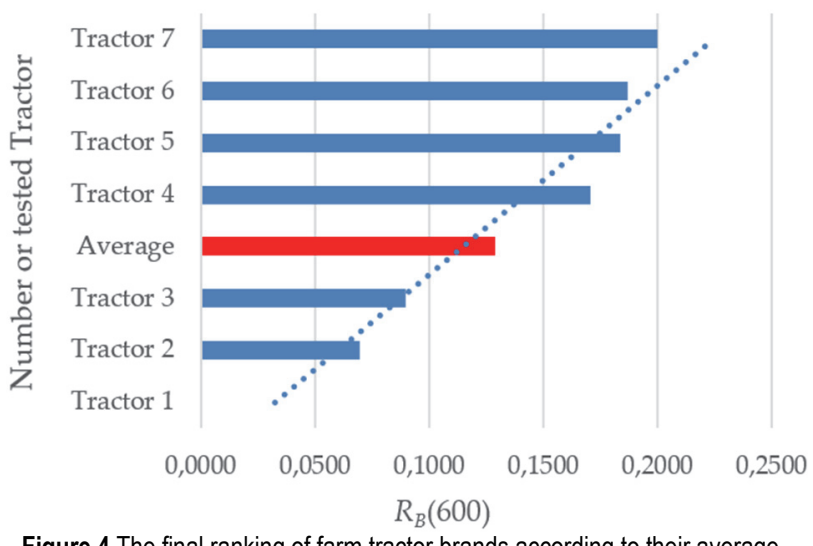

Figure 4 The final ranking of farm tractor brands according to their average reliability $R_{B}(600)$ and the trend line

The calculations were made for $\Delta t=600$ hours of operation, i.e. the approximate average annual time of operation of a tractor in Poland. As the chart shows, Massey Ferguson tractors made by the CanadianAmerican agricultural machinery manufacturer were ranked as the most reliable $R_{\mathrm{MF}}(600)=0.2000$ of all the tractors rated in the survey (Fig. 5).

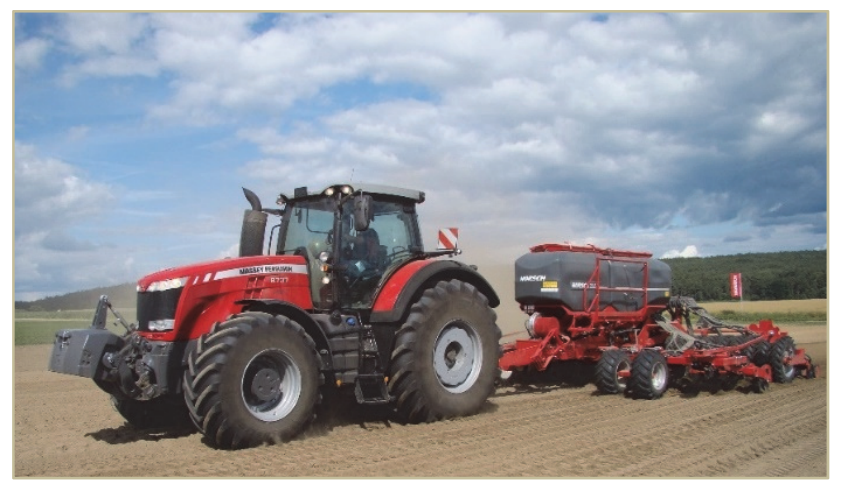

Figure 5 Massey Ferguson brand tractor, winner of the reliability ranking HorschMaschinen GmbH, Schwandorf, Germany

Table 1 The empirical data on the number and type of failures of farm tractors and the results of partial calculations

\begin{tabular}{|c|c|c|c|c|c|c|c|c|c|c|c|}
\hline No. & $\begin{array}{l}\text { Brand/ } \\
\text { model }\end{array}$ & $\begin{array}{c}\Delta t \\
\text { hours of } \\
\text { operation }\end{array}$ & $\begin{array}{c}F_{r} \\
\text { number }\end{array}$ & $\begin{array}{c}F_{a} \\
\text { number }\end{array}$ & $\begin{array}{c}\lambda_{r} \\
\text { hours of }^{\text {operation }}{ }^{-1}\end{array}$ & $\begin{array}{c}\lambda_{a} \\
\text { hours of } \\
\text { operation }^{-1}\end{array}$ & $\lambda_{r} / \lambda_{a}$ & $\exp (x)$ & $\operatorname{erfc}(x)$ & $P(E \leq W)$ & $\begin{array}{c}\text { Type of } \\
\text { distribution }\end{array}$ \\
\hline 1. & 2 & 3 & 4 & 5 & 6 & 7 & 8 & 9 & 10 & 11 & 12 \\
\hline 2. & Belarus 82 & 100 & 22 & 0 & 0.2200 & 0 & - & - & - & 1.00 & $\mathrm{E}$ \\
\hline 3. & Belarus 1025a & 2200 & 1 & 7 & 0.0005 & 0.0032 & 0.1429 & 1.0051 & 0.9195 & 0.12 & $\mathrm{~W}$ \\
\hline 4. & Belarus MTZ 82 & 2000 & 8 & 16 & 0.0400 & 0.0080 & 0.5000 & 1.0645 & 0.737 & 0.34 & $\mathrm{~W}$ \\
\hline 5. & Case IH MX 170 & 654 & 2 & 1 & 0.0031 & 0.0015 & 2.0000 & 2.7183 & 0.1573 & 0.76 & $\mathrm{E}$ \\
\hline 6. & Case IH MX 170 & 22442 & 12 & 59 & 0.0005 & 0.0026 & 0.2034 & 1.0104 & 0.8856 & 0.16 & $\mathrm{~W}$ \\
\hline & ( & & & & & & & & & & \\
\hline 25. & Zetor 5320 & 1500 & 5 & 11 & 0.0033 & 0.0073 & 0.4028 & 1.0530 & 0.7479 & 0.32 & $\mathrm{~W}$ \\
\hline 26. & Zetor Major 7340 & 6499 & 16 & 14 & 0.0025 & 0.0022 & 1.1429 & 1.3862 & 0.4190 & 0.59 & $\mathrm{E}$ \\
\hline 27. & Zetor Proxima 100 Power & 1893 & 15 & 7 & 0.0079 & 0.0037 & 2.1429 & 3.1518 & 0.1297 & 0.78 & $\mathrm{E}$ \\
\hline
\end{tabular}

\section{SUMMARY AND CONCLUSIONS}

The results confirmed the good reputation of Massey Ferguson. In 2020, which was a hard year, this brand of tractors sold the best in Lithuania (123 tractors), whereas in Norway and Sweden it was the third best-selling brand. The quality and reliability of Massey Ferguson tractors were also appreciated by the jurors of the prestigious 
Tractor of the Year 2021 competition, who gave the award to the Massey Ferguson tractor model 8S.265. The jury assessed the following parameters: engine, gearbox, standard electronic devices facilitating tractor operation, hydraulic systems, cabin comfort, technological innovations, options, design, communication and precision farming. The Massey Ferguson tractor beat other brands such as: Armatrac, Case, Claas, Fendt, Kubota, Landini. New Holland, and Steyr.

Although NH tractors are a budget brand (also above the average of 0.13 ), Polish farmers have positive opinions about them. It is the third most popular brand in Croatia. Of the total number of 981 tractors sold in 202077 were Massey Ferguson products. Only John Deere (115 tractors) and Carraro (79 tractors) sold more products.

There were two other well-known brands ranked above average: the American Case IH and the ItalianGerman concern SAMEDeutz-Fahr SDF. The following brands were ranked the lowest: Minsk Tractor Factory MTZ Belarus and Zetor Tractors a.s. the Czech manufacturer of farm tractors. The users found a large number of failures, especially random ones. In consequence, they had lower values of the indicator $R(\Delta t)$.

It should not be surprising that after only one year of operation there were such low values of reliability indicators. By assumption, both the one-parameter exponential distribution and the two-parameter Weibull distribution are absolute. Even individual failures dramatically reduce the reliability of technical devices.

Classic scientific validation is not possible here because there are no other rankings of the $R_{B}(t)$ parameter in the available domestic and foreign publications.

The use of farm tractors is a continuous process in time and discrete in the states. It takes place in specific and often extremely different terrain and weather conditions. The reliability of farm tractors definitely affects the efficiency of work on a farm which involves cyclical and interrelated machine operations. It is difficult to convert reliability into a numerical form that will take all parameters of the tested facility into account. This task is practically impossible because manufacturers do not provide detailed service histories. However, it is possible to use the classic method of quantification of machine reliability by estimating its level.

The method was based on the well-known reliability theory supplemented with the method of acquisition of reliable data. The a priori assumption was that users would not provide comprehensive service information about the types of failures. Due to the incompleteness of this information, it was necessary to make well thought-out simplifications and assumptions, which did not distort the order of brands in the rankings.

The simulation tests led to the following conclusions: 1. The competing risks method invented by Daniel Bernoulli enables clear indication of the best type of distribution to quantify the reliability of farm tractors based on the probability $P(E \leq W)$. If age-related failures predominate, the two-parameter Weibull distribution should be used. If random failures predominate, the oneparameter exponential function is better.

2. The proposed method makes use of both of these statistical distributions to quantify the operational reliability of farm tractors in the form of a relative $R_{B}(t)$ indicator of a particular brand. The method is logically correct. Agricultural engineering experts' and farmers' opinions about the reliable operation have also been confirmed. Thus, the method is also empirically verifiable. 3. In comparison with the classical approach, which requires precise data on failure occurrence times, the competing risks method is a simplification. Failure occurrence times make it possible to determine the average durability of a technical device, whereas the inverse gives a failure intensity indicator.

4. The process of quick quantification of the reliability of farm tractors supports operational research, because it develops methods which support the decisionmaking process to minimize uncertainty and risk. Thus, the method can also be used in practice to quantify the reliability of other technical devices. It is only necessary to acquire objective and extensive data on the number of random and age-related failures from a large group of reliable users. This considerably simplifies the reliability quantification method. There is no need to provide a detailed service history, which is usually a significant problem (e.g. no maintenance log). It is enough to know the number of failures (and the number of repairs) of a particular tractor due to sudden and gradual failures occurring during operation.

5. The advantage of the method is that there is no need to build a reliability model of a technical device (tractor) because it assumes that all tractor components are equally important and serially connected.

6 . The disadvantage of the method might be the problem to classify a particular failure as a random or agerelated one. Respondents would need to have extensive theoretical and practical knowledge.

7. The popularization of the method and the development of an Internet application for collecting information about farm tractor failures will enable the development of more detailed reliability rankings in the future. These new reliability rankings will include not only the brand, but also the model, engine power, year of manufacture, as well as individual components of tractors. 8. Data on the number of random and age-related failures of the working components of tractors of different brands provided by long-term users over a period of time are a valuable and desirable source of information not only for potential customers who need to choose one out of more than 50 brands available on the market, but also for manufacturers who can verify the frequency of periodic overhauls and warranty conditions, as well as sellers and servicemen.

\section{Acknowledgments}

This research was funded by Poznan University of Technology, grant number 0414/SBAD/3612.

\section{REFERENCES}

[1] Poozesh, M., Mohtasebi, S., \& Ahmadi, H. (2011). Determining the reliability function of farm tractors. 11th International Congress on Mechanization and Energy in Agriculture (TRAKAGENG-2011). Retrieved from https://www.researchgate.net/publication/236679761

[2] Bedford, T., Quigley, J., Walls L., Alkali, B., Daneshkhah, A., \& Hardman, G. (2008). Advances in mathematical 
modeling for reliability. Amsterdam. IOS Press. https://doi.org/10.1002/9780470061572.eqr352

[3] Kanchan, D. \& Walid, A. K. (2011). Consideration of dynamic changes in machine reliability and part demand: a cellular manufacturing systems design model. International Journal of Production Research, 49(7), 2123-2142. https://doi.org/10.1080/00207541003641879

[4] Linqvist, B. H. \&Doksun, K. A. (2003). Mathematical and Statistical Methods in Reliability. Series on Quality, Reliability and Engineering Statistics, 7. World Scientific Publishing Co. Pte. Ltd. https://doi.org/10.1142/5248

[5] Pham, H. (2006). Reliability modeling, analysis and optimization. Series on Quality. Reliability and Engineering Statistics, 9. World Scientific Publishing Co. Pte. Ltd. https://doi.org/10.1007/1-84628-295-0 5

[6] Farrington, C. P., Kanaan, M. N., \& Gay, N. J. (2001). Estimation of the basic reproduction numberfor infectious diseases from age-stratified serological survey data. Applied Statistics, 50(3), 251-292. https://doi.org/10.1111/1467-9876.00233

[7] Dietz, K. \& Heesterbeek J. A. P. (2002). Daniel Bernoulli's epidemiological model revisited. Mathematical Biosciences, 180, 1-21. https://doi.org/10.1016/S0025-5564(02)00122-0

[8] Fine, F. \& Lindqvist, B. H. (2014). Competing risks. Lifetime Data Analysis, 20(2), 159-160. https://doi.org/10.1007/s10985-014-9294-8

[9] Andrzejczak, K. \& Popowska, B. (2011). Three parameter model of the system lifetime with two types of risks. Maintenance Problems, 80, 17-24. https://doi.org/10.1007/s10985-010-9169-6

[10] Bakoyannis, G. \& Touloumi, G. (2012). Practical methods for competing risks data: a review. Statistical Methods in Medical Research, 21(3), 257-272. https://doi.org/10.1177/0962280210394479

[11] Lee, C., Yoon, J., \& Schaar M. (2020). Dynamic-DeepHit: A Deep Learning Approach for Dynamic Survival Analysis With Competing Risks Based on Longitudinal Data. IEEE Transactions on Biomedical Engineering, 67(1), 122-133, https://doi.org/10.1109/TBME.2019.2909027

[12] Satagopan, J. M., Ben-Porat, L., Berwick, M., Robson, M., Kutler D., \& Auerbach A. D. (2004). A note on competing risks in survival data analysis. British Journal of Cancer, 91(7), 1229-1235. https://doi.org/10.1038/sj.bjc.6602102

[13] Somboonsavatdee, A. \& Sen, A. (2015). Statistical Inference for Power-Law Process With Competing Risks, Technometrics, 57(1), 112-122. https://doi.org/10.1080/00401706.2014.902772

[14] Tan, F., Hou, X., Zhang, J., Wei, Z. \&Yan, Z. (2019). A Deep Learning Approach to Competing Risks Representation in Peer-to-Peer Lending. IEEE Transactions on Neural Networks and Learning Systems, 30(5), 1565-1574. https://doi.org/10.1109/TNNLS.2018.2870573

[15] Wang, Y., Shi, Y., \& Wu, M. (2019). Statistical inference for dependence competing risks model under middle censoring. Journal of Systems Engineering and Electronics, 30(1), 209222. https://doi.org/10.21629/JSEE.2019.01.20

[16] Craiu, R. V., \& Lee T. C. (2005). Model Selection for the Competing-Risks Model With and Without Masking. Technometrics, 47(4), 457-467. https://doi.org/10.1198/004017005000000238

[17] Crowder, M. J. (2001). Classical competing risks. New York, Chapman \& Hall/CRC. https://doi.org/10.1201/9781420035902

[18] Mendenhall, W. \& Hader R. (1958). Estimation of parameters of mixed exponentially distributed failure time distributions from censored life test data. Biometrika, 45(34), 504-520. https://doi.org/10.2307/2333197

[19] Cooke, R. M. (1996). The design of reliability databases, Part I: review of standard design concepts. Reliability
Engineering and System Safety, 51(2), 137-146. https://doi.org/10.1016/0951-8320(95)00111-5

[20] Durczak, K., Ekielski, A., Kozłowski, R., Żelaziński, T., \& Pilarski, K. (2020). A computer system supporting agricultural machinery and farm tractor purchase decisions. Heliyon, 6(10), https://doi.org/10.1016/j.heliyon.2020.e05039

[21] Jurek, P. (2019). A System for Quantifying the Reliability of Agricultural Machinery and Vehicles. Doctoral dissertation written at the Department of Biosystem Engineering, Faculty of Agronomy and Bioengineering, Poznań University of Life Sciences.

[22] Durczak, K. (2020). Reliability of agricultural tractors according to Polish farmers. Technical Gazette, 27/6, 17601765. https://doi.org/10.17559/TV-20190819132340

[23] Audsley, E. (1999). Operational Research, Agriculture and the Environment. The Journal of Agricultural Science, 133(4), 455-458. https://doi.org/10.1017/S0021859699007224

[24] Aven, T. (2008). Risk Analysis. New York: WILEY. https://doi.org/10.1002/9780470694435

[25] Greco, S., Ehrgott, M., \& Figueira J. R. (2016). Multiple Criteria Decision Analysis. State of the Art Surveys, International Series in Operations Research \& Management Science, 233, New York, Springer Science+Bisness Media. https://doi.org/10.1007/978-1-4939-3094-4

[26] Majumder, M. (2015). Impact of Urbanization on Water Shortage in Face of Climatic Aberrations. Springer Briefs in Water Science and Technology. https://doi.org/10.1007/978-981-4560-73-3_2

[27] Miettinen, K. (1999). Nonlinear Multiobjective Optimization. Boston, Kluwer. https://doi.org/10.1007/978-1-4615-5563-6

[28] Bouyssou, D. \& Roy, B. (1993). Aide Multicritere a la Decision: Methodes at Cas., Paris,Economica.

[29] Tohidi, A., Ghorbani, M., Karbasi, A. R., Asgharpourmasouleh, A., \& Hassani-Mahmooei, B. (2020). Prioritization of Business Strategies and Marketing Resources Using the Analytic Network Process (ANP) Approach. Journal of Agricultural Science and Technology, 22(3), 611-624. Retrieved from https://www.researchgate.net/publication/340922980

\section{Contact information:}

Karol DURCZAK, Assoc. Prof. PULS

(Corresponding author)

Department of Biosystems Engineering,

Faculty of Environmental and Mechanical Engineering,

Poznań University of Life Sciences,

UI. WojskaPolskiego 50, 60-627 Poznań, Poland

E-mail: karol.durczak@up.poznan.pl

Jaroslaw SELECH, Assoc. Prof.

Poznan University of Technology,

5 M. Skłodowska-Curie Square PL-60-965 Poznań, Poland

E-mail: jaroslaw.selech@put.poznan.pl 